\title{
The Hough Transform without the Accumulators
}

\author{
Atsushi Imiya ${ }^{1,2}$, Tetsu Hada ${ }^{2}$, and Ken Tatara ${ }^{2}$ \\ 1 National Institute of Informatics \\ 2-1-2 Hitotsubashi, Chiyoda-ku, Tokyo 101-8430 \\ 2 IMIT, Chiba University \\ 1-33 Yayoi-cho, Inage-ku, 263-8522, Chiba, Japan \\ imiya@media.imit.chiba-u.ac.jp \\ imiya@nii.ac.jp
}

\begin{abstract}
The least-squares method (LSM) efficiently solves the modelfitting problem, if we assume a model equation. For the fitting to a collection of models, the classification of data is required as pre-processing. The Hough transform, achieves both the classification of sample points and the model fitting concurrently. However, as far as adopting the voting process is concerned, the maintenance of the accumulator during the computation cannot be neglected. We propose a Hough transform without the accumulator expressing the classification of data for the model fitting problems as the permutation of matrices which are defined by data.
\end{abstract}

\section{Introduction}

The Hough transform simultaneously detects many conics on a plane. The basic idea of the Hough transform is the classification of sample points and identification of parameters of figures by voting dual figures to the accumulator space $[1,2,3,4]$. For the achievement of the Hough transform, the maintenance of dual figures in the accumulator space is a fundamental task. For the maintenance of the accumulator space, we are required to prepare large memory areas. Therefore, to derive a simple method for the detection of many conics in a plane, we, in this paper introduce the Hough transform without the accumulator space. The method is based on the property that the classification of sample points is achieved by the permutation of a sequence of sample points and and the matrix representation of a permutation defines a zero-one orthogonal matrix. Furthermore, once the classification of sample points is established, the estimation of parameters of conics is achieved by solving the least-mean-squares criterion. This second process is achieved by the eigenvalue decomposition of the moment matrix constructed sample points.

The eigenvalue decomposition is established by computing orthogonal matrix which diagonalizes the moment matrix. We first define the minimization criterion for the Hough transform. Second, we derive a dynamic system which guarantees the convergence of the Hough transform. Finally, we derive a relaxation method to solve the criterion for the Hough transform without using any accumulator spaces. We apply the method for the detection of conics on a plane. 


\section{Mathematical Aspects of the Hough Transform}

\subsection{Figures and Dual Figures}

Setting $\boldsymbol{x}=(x, y)^{\top}$ to be a vector in two-dimensional Euclidean space $\mathbf{R}^{2}$, we define the homogeneous coordinate $\boldsymbol{\xi}$ of a vector $\boldsymbol{x} \in \mathbf{R}^{k}$ for $k \geq 3$ as $\boldsymbol{\xi}=\left(\boldsymbol{x}^{\top}, 1\right)^{\top}$. We denote the Euclidean length of vector $\boldsymbol{x}$ in $k$-dimensional Euclidean space $\mathbf{R}^{k}$ for $k \geq 1$ as $|\boldsymbol{a}|$. Let $S^{n-1}$ be the unit sphere of $\mathbf{R}^{n}$ consisting of all points $\boldsymbol{x}$ with distance 1 from the origin. For $n=1, S^{0}=[-1,1]$. Furthermore, the positive half-space is defined by $\mathbf{R}_{+}^{n}=\left\{\boldsymbol{x} \mid x_{n}>0\right\}$, for $n \geq 1$. Now, setting $H_{+}^{n-1}=S^{n-1} \cap \mathbf{R}_{+}^{n}$, for $n \geq 1$, the positive unit semi-sphere is defined as $S_{+}^{n-1}=S_{+}^{n-2} \bigcup H_{+}^{n-1}$, for $n \geq 1$.

Let $\mathcal{P}_{2}^{k}=\left\{p_{i}(x, y)\right\}_{i=1}^{k}$ be a set of independent monomials of $x$ and $y$, and let $\mathcal{A}^{k}=\left\{\boldsymbol{a} \mid \boldsymbol{a}=\left\{a_{i}\right\}_{i=1}^{k}\right\}$ be a set of all $n$-tuple real numbers, where at least one of $a_{i}$ is nonzero. Then, setting $P(x, y)=\sum_{i=1}^{k} a_{i} p_{i}(x, y)$, a set $\mathcal{C}_{2}\left(\mathcal{P}_{2}^{k}, \mathcal{A}^{k}\right)=$ $\left\{(x, y)^{\top} \mid P(x, y)=0\right\}$ defines a family of curves on the plane for $\boldsymbol{a} \in \mathcal{A}^{n}[5]$. Here, the suffix 2 of $\mathcal{C}_{2}\left(\mathcal{P}_{2}^{n}, \mathcal{A}^{k}\right)$ indicates a set of algebraic curves of two real arguments. The following theorems are held.

Theorem 1 An element of $\mathcal{A}^{k}$ corresponds to a point in the $n$-dimensional vector space.

From theorem 1, we define a coefficient vector of $P(x, y)$ as $\boldsymbol{a}=$ $\left(a_{1}, a_{2}, \cdots, a_{k}\right)^{\top}$. For a positive real value $\lambda, \lambda P(x, y)=0$ and $-\lambda P(x, y)=0$ define the same curve. Conversely, once a point $\boldsymbol{a}$ on $S_{+}^{k-1}$ is fixed, we obtain a curve of $\mathcal{C}_{2}\left(\mathcal{P}_{2}^{k}, \mathcal{A}^{k}\right)$. This leads to the following theorem.

Theorem 2 There is one-to-one mapping between $\mathcal{C}_{2}\left(\mathcal{P}_{2}^{k}, \mathcal{A}^{n}\right)$ and $S_{+}^{k-1}$.

If elements of $\mathcal{P}_{2}^{6}$ are $p_{1}(x, y)=1, p_{2}(x, y)=2 x, p_{3}(x, y)=2 y, p_{4}(x, y)=x^{2}$, $p_{5}(x, y)=2 x y$, and $p_{6}(x, y)=y^{2}$, we obtain

$$
P(x, y)=a x^{2}+2 b x y+c y^{2}+2 d x+2 e y+f .
$$

Then, $\mathcal{C}_{2}\left(\mathcal{P}_{2}^{6}, \mathcal{A}^{6}\right)$ determines the family of conics in the Euclidean plane if at least one of $a, b$, and $c$, and $f$ are not zero. Therefore, the Hough transform is a method for the detection of points on $S_{+}^{5}$, which coresspond to conics, from noisy samples on the image in plane. We call a point on $S_{+}^{k-1}$ the dual figure of a curve on $\mathbf{R}^{2}$.

\subsection{The Hough Transform as LMS}

Let $m$ conics exist on $\mathbf{R}^{2}$ and sample-points $P$ be separated to clusters of points as $P=\bigcup_{i=1}^{m} P_{i}$ such that $P_{i}=\left\{\boldsymbol{x}_{i j}\right\}_{j=1}^{n(i)}$. Assuming that points in each cluster 
distribute in the neighborhood of conics, the conic fitting problem for a collection of conics is achieved minimizing the functional

$$
J\left(\boldsymbol{a}_{1}, \cdots, \boldsymbol{a}_{m}\right)=\sum_{i=1}^{m}\left\{\sum_{j=1}^{n(i)} w_{i}(j)\left(\boldsymbol{\xi}_{i j}^{\top} \boldsymbol{a}_{i}\right)^{2}-\lambda_{i}\left(1-\left|\boldsymbol{a}_{i}\right|^{2}\right)\right\},
$$

where $w_{i}(j) \geq 0$ and $\sum_{i=1}^{m} \sum_{j=1}^{n(i)} w_{i}(j)=1$. This minimization problem yields a system of eigenvalue equations

$$
\boldsymbol{M}_{i} \boldsymbol{a}_{i}=\lambda_{i} \boldsymbol{a}_{i}, \text { s.t. } \boldsymbol{M}_{i}=\sum_{j=1}^{n(i)} w_{i}(j) \boldsymbol{\xi}_{i j} \boldsymbol{\xi}_{i j}^{\top}
$$

for $i=1,2, \cdots, m$. Furthermore, the solutions are the eigenvectors which associate to the smallest eigenvalues of each problem. In this paper, we assume that both $w(i)$ and $w_{i}(j)$ are $1 / n$. Furthermore, we assume that in each cluster there exists $k$ points, that, is $k=n(i)$ and $k \times m=n$.

The Hough transform for the conic detection achieves both the classification of sample points and the model fitting concurrently. Therefore, in this section, we formulate the Hough transform as the LSM for the model fitting problem. Setting $\boldsymbol{\Xi}=\left(\boldsymbol{\xi}_{1}, \boldsymbol{\xi}_{2}, \cdots, \boldsymbol{\xi}_{n}\right)^{\top}$, if there is no error in sample points, the parameters of a conic satisfies the equation $\boldsymbol{\Xi}^{\top} \boldsymbol{a}=\mathbf{0}$. For the noisy data, the parameter of a conic is the solution of the functional

$$
J(\boldsymbol{a})=\left|\boldsymbol{\Xi}^{\top} \boldsymbol{a}\right|^{2}-\lambda\left(1-|\boldsymbol{a}|^{2}\right) .
$$

If there exist $m$ conics, after clustering sample-points, we have the equation, $\boldsymbol{\Xi}_{i}^{\top} \boldsymbol{a}_{i}=\mathbf{0}$, for $i=1,2, \cdots m$, where $\boldsymbol{\Xi}=\left(\boldsymbol{\xi}_{i(1)}, \boldsymbol{\xi}_{i(2)}, \cdots, \boldsymbol{\xi}_{i(k)}\right)$. If we do not know any clusters of sample-points, the minimization criterion is expressed as $(\boldsymbol{Q} \overline{\boldsymbol{\xi}})^{\top} \overline{\boldsymbol{a}}=0$, where $\boldsymbol{Q}$ is an appropriate permutation matrix and, $\overline{\boldsymbol{\xi}}=\left(\boldsymbol{\xi}_{1}^{\top}, \boldsymbol{\xi}_{2}^{\top}, \cdots, \boldsymbol{\xi}_{n}^{\top}\right)^{\top}, \overline{\boldsymbol{a}}=\left(\overline{\boldsymbol{a}}_{1}^{\top}, \overline{\boldsymbol{a}}_{2}^{\top}, \cdots, \overline{\boldsymbol{a}}_{m}^{\top}\right)^{\top}$, and $\overline{\boldsymbol{a}}_{i}=(\underbrace{\boldsymbol{a}_{i}^{\top}, \boldsymbol{a}_{i}^{\top}, \cdots, \boldsymbol{a}_{i}^{\top}}_{k^{\prime} s})^{\top}$.

Therefore, the parameters minimize the functional

$$
J(\boldsymbol{a}, \boldsymbol{Q})=\left|(\boldsymbol{Q} \overline{\boldsymbol{\xi}})^{\top} \overline{\boldsymbol{a}}\right|^{2}-\sum_{i=1}^{m} \lambda_{i}\left(1-\left|\boldsymbol{a}_{i}\right|^{2}\right) .
$$

This property implies that the classification of sample data is equivalent to the permutation of elements of $\boldsymbol{\Xi}$. There exist many possibilities for the selection of a permutation $\boldsymbol{Q}$, if we do not know the estimate of $\left\{\boldsymbol{a}_{i}\right\}_{i=1}^{m}$ [6]. These expressions of the conic fitting problem conclude that the Hough transform achieves both the permutation of data matrix $\boldsymbol{\Xi}$ and the computation of the parameters of conics concurrently.

\subsection{Dyanamics of the Hough Transform}

We consider the problem to determine a $r$-dimensional linear subspace in $\mathbf{R}^{n}$, where $1<r<n$ which approximate the distribution of data-set $\left\{\boldsymbol{y}_{i}\right\}_{i=1}^{k}$, the 
mean of which are zero, that is, $\sum_{i=1}^{k} \boldsymbol{y}_{i}=0$. The orthogonal projection matrix $\boldsymbol{P}$ such that $\operatorname{rank} \boldsymbol{P}=r$ which minimizes the criterion $\varepsilon^{2}=\operatorname{tr}(\boldsymbol{P} \boldsymbol{M})$, for $\boldsymbol{M}=$ $\frac{1}{k} \sum_{i=1}^{k} \boldsymbol{y}_{i} \boldsymbol{y}_{i}^{\top}$, determines the $r$-dimensional linear subspace which approximate the distribution of $\left\{\boldsymbol{y}_{i}\right\}_{i=1}^{k}$.

Since matrices $\boldsymbol{P}$ and $\boldsymbol{M}$ are symmetry matrices, there exist orthogonal matrices $\boldsymbol{U}$ and $\boldsymbol{V}$ which diagonalize $\boldsymbol{P}$ and $\boldsymbol{M}$, respectively. If we set $\boldsymbol{P}=$ $\boldsymbol{U} \boldsymbol{\Lambda} \boldsymbol{U}^{\top}$ and $\boldsymbol{M}=\boldsymbol{V} \boldsymbol{D} \boldsymbol{V}^{\top}$, where $\boldsymbol{\Lambda}$ is a diagonal matrix whose entries are 0 or 1 such that $\operatorname{tr} \boldsymbol{\Lambda}=r$, and $\boldsymbol{D}$ is a diagonal matrix. These decomposition of matrices derive $\varepsilon^{2}=\operatorname{tr}\left(\boldsymbol{W} \boldsymbol{\Lambda} \boldsymbol{W}^{\top} \boldsymbol{D}\right)$, where $\boldsymbol{W}=\boldsymbol{V}^{\top} \boldsymbol{U}$, since $\operatorname{tr}(\boldsymbol{P} \boldsymbol{M})=$ $\operatorname{tr}\left(\boldsymbol{U} \boldsymbol{\Lambda} \boldsymbol{U}^{\top} \boldsymbol{V} \boldsymbol{D} \boldsymbol{V}^{\top}\right)=\operatorname{tr}\left(\boldsymbol{V}^{\top} \boldsymbol{U} \boldsymbol{\Lambda} \boldsymbol{U}^{\top} \boldsymbol{V} \boldsymbol{D}\right)$. Therefore, our problem is mathematically equivalent to the determination of an orthogonal matrix which minimizes the criterion for the detection of conics from unclusterd data.

The gradient flow for $\boldsymbol{W}$

$$
\frac{d}{d t} \boldsymbol{W}=-\left[\boldsymbol{\Lambda}, \boldsymbol{W} \boldsymbol{D} \boldsymbol{W}^{\top}\right] \boldsymbol{W}
$$

where $[\boldsymbol{X}, \boldsymbol{Y}]=\boldsymbol{X} \boldsymbol{Y}-\boldsymbol{Y} \boldsymbol{X}$, is the continuous version of the gradient decent equation to search an orthogonal matrix which minimizes the criterion $[7,8]$ for the detection of conics from unclustered data. Furthermore, setting $\boldsymbol{G}=$ $\boldsymbol{W} \boldsymbol{\Lambda} \boldsymbol{W}^{\top}$, eq. (6) is equivalent to the double bracket equation,

$$
\frac{d}{d t} \boldsymbol{G}=-[\boldsymbol{G},[\boldsymbol{G}, \boldsymbol{D}]]
$$

If $n=6$ and $r=1$, our equation determines a conic on a plane using the homogeneous coordinate system. For the detection of many conics on a plane, the criterion for the minimization is $\varepsilon^{2}=\left|(\boldsymbol{Q} \overline{\boldsymbol{\xi}})^{\top} \overline{\boldsymbol{a}}\right|^{2}=\operatorname{tr}\left(\boldsymbol{A} \boldsymbol{Q} \boldsymbol{M} \boldsymbol{Q}^{\top}\right)$, where $\boldsymbol{A}=\overline{\boldsymbol{a}} \overline{\boldsymbol{a}}^{\top}$, and $\boldsymbol{M}=\overline{\boldsymbol{\xi}} \overline{\boldsymbol{\xi}}^{\top}$. Let $\Theta$ be an orthogonal matrix which digonalizes $\boldsymbol{A}$ such that $\boldsymbol{A}=\boldsymbol{\Theta}(\boldsymbol{I} \otimes \operatorname{Diag}(1,0,0,0,0,0)) \boldsymbol{\Theta}^{\top}$. Since $\boldsymbol{A}$ is the covariance matrix, matrix $\boldsymbol{A}$ is expressed as the Kroneker product of the identity matrix and $\operatorname{Diag}(1,0,0,0,0,0)$ using an appropriate coordinate system, where $m$ is the number of conics which exist on a plane. The minimization of $\epsilon^{2}$ is equivalent to minimize $\operatorname{tr}\left(\boldsymbol{Q} \boldsymbol{U} \boldsymbol{\Lambda} \boldsymbol{U}^{\top} \boldsymbol{Q}^{\top} \boldsymbol{\Theta} \boldsymbol{D} \boldsymbol{\Theta}^{\top}\right)=\operatorname{tr}\left(\boldsymbol{W} \boldsymbol{\Lambda} \boldsymbol{W}^{\top} \boldsymbol{D}\right)$, if we set $\boldsymbol{D}=\boldsymbol{I} \otimes \operatorname{Diag}(1,0,0,0,0,0)$ and $\boldsymbol{W}=\boldsymbol{\Theta}^{\top} \boldsymbol{Q} \boldsymbol{U}$. This expression of the criterion for the detection of many conics implies that the conic detection trough the Hough transform is also achieved by the gradient flow.

The double bracket dynamics has clarified mathematical property of the Hough transform which concurrently detects many conics on a plane from noisy data. This dynamics implies that the permutation which is described as a orthogonal matrix achieves grouping of data for the detection of conics. Furthermore, this dynamics implies that if we can generate a decresing sequence with respect to the energy function $\operatorname{tr}\left(\boldsymbol{A} \boldsymbol{Q} \boldsymbol{M} \boldsymbol{Q}^{\top}\right)$, we can compute the minimum value of the criterion.

For $\operatorname{tr} \boldsymbol{\Lambda}=r$, we have the relation $\operatorname{tr}\left(\boldsymbol{I} \boldsymbol{U} \boldsymbol{P} \boldsymbol{U}^{\top}\right)=\operatorname{tr}(\boldsymbol{I} \boldsymbol{P})=\operatorname{tr} \boldsymbol{P}$. Therefore, projection matrix $\boldsymbol{P}$ is defined as the solution of a semidefinite programming 
problem. From the orthogonal decomposition of projection matrix $\boldsymbol{P}$ and moment matrix $\boldsymbol{M}$, we have $\operatorname{tr}(\boldsymbol{I} \boldsymbol{A})=m$ if the number of conics is $m$. Therefore, the detection of conics is achieved by minimizing $\operatorname{tr}\left(\boldsymbol{Q}^{\top} \boldsymbol{A} \boldsymbol{Q} \boldsymbol{M}\right)$ subject to $\operatorname{tr}(\boldsymbol{I} \boldsymbol{A})=m$.

\section{Combinatorial Hough Transform}

Vectors $\left\{\boldsymbol{a}_{i}^{*}\right\}_{i=1}^{m}$ and matrix $\boldsymbol{Q}^{*}$, which minimize $J\left(\boldsymbol{a}_{1}, \boldsymbol{a}_{2}, \cdots, \boldsymbol{a}_{m}, \boldsymbol{Q}\right)=$ $\left|\overline{\boldsymbol{\Xi}}^{\top} \overline{\boldsymbol{Q}} \boldsymbol{a}\right|^{2}$, w.r.t. $\left|\boldsymbol{a}_{i}\right|=1$, determine conics which fit to sample points $\left\{\boldsymbol{x}_{i}\right\}_{i=1}^{m}$. It is, however, not so easy to determine all vectors $\boldsymbol{a}_{i}$, for $i=1,2, \cdots, m$ and matrix $\boldsymbol{Q}$, simultaneously. If $\boldsymbol{Q}$ is determined, $\left\{\boldsymbol{a}_{i}\right\}_{i=1}^{m}$ is computed using semidefinite programming. Furthermore, if the set of parameters $\left\{\boldsymbol{a}_{i}\right\}_{i=1}^{m}$ is determined, $\boldsymbol{Q}$ is computed using integer programming. Using the relaxation, for $\left\{\boldsymbol{a}_{i}\right\}_{i=1}^{n}$ and $\boldsymbol{Q}$, we compute the minimum of $J\left(\boldsymbol{a}_{1}, \boldsymbol{a}_{2}, \cdots, \boldsymbol{a}_{m}, \boldsymbol{Q}\right)$ as follows.

\section{Algorithm 1}

1. Set initial estimation of $\boldsymbol{Q}$ as $\boldsymbol{Q}_{0}$.

2. Compute $\boldsymbol{a}_{i}^{*} i=1,2, \cdots, m$, which minimize $J\left(\boldsymbol{a}_{1}, \boldsymbol{a}_{2}, \cdots, \boldsymbol{a}_{m}, \boldsymbol{Q}_{0}\right)$.

3. Compute $\boldsymbol{Q}^{*}$ which minimizes $J\left(\boldsymbol{a}_{1}^{*}, \boldsymbol{a}_{2}^{*}, \cdots, \boldsymbol{a}_{m}^{*}, \boldsymbol{Q}\right)$ and satisfies the inequality such that $J\left(\boldsymbol{a}_{1}^{*}, \boldsymbol{a}_{2}^{*}, \cdots, \boldsymbol{a}_{m}^{*}, \boldsymbol{Q}^{*}\right)<J\left(\boldsymbol{a}_{1}^{*}, \boldsymbol{a}_{2}^{*}, \cdots, \boldsymbol{a}_{m}^{*}, \boldsymbol{Q}_{0}\right)$.

4. If $\boldsymbol{Q}_{0} \neq \boldsymbol{Q}^{*}$ then $\boldsymbol{Q}_{0}:=\boldsymbol{Q}^{*}$ and go to 2 else if $J\left(\boldsymbol{a}_{1}^{*}, \boldsymbol{a}_{2}^{*}, \cdots, \boldsymbol{a}_{m}^{*}, \boldsymbol{Q}^{*}\right)>\varepsilon$, for a small positive constant $\varepsilon$, then go to 1 else accept $\boldsymbol{a}_{i}^{*}$, for $i=1,2, \cdots, m$ as the solutions.

5. Draw conics $\boldsymbol{\xi}^{\top} \boldsymbol{a}^{*}=0$, for $i=1,2, \cdots, m$.

Here, $\varepsilon \leq m \delta$, where $\delta$ is introduced in the next section using the discretization method of conics. We call this process the combinatorial Hough transform. Step 2 is achieved by semidefinite programming $[9,10]$ and step 3 is solved by integer programming. In this paper, for step 3 , we define a new matrix $Q^{*}$ computing the distance between sample points and detected figures. If both $\boldsymbol{Q}^{*}$ and $\boldsymbol{a}_{k}^{*}$ are correct ones, the total sum of the distances between points and figures become minimum. For the evaluation of these distances, we compute the minimum distance from each sample point to figures. This process determines a $\boldsymbol{Q}^{*}$ which satisfies the condition of step 3 .

Employing the marge sort, and divide and conquer methods, we divide the array of sample points into subsets. Dividing both intervals $I(x)=\left\{x \mid-\frac{L}{2} \leq x \leq\right.$ $\left.\frac{L}{2}\right\}$ and $I(y)=\left\{v \mid-\frac{L}{2} \leq y \leq \frac{L}{2}\right\}$, into $k$ subintervals equally as $\cup_{i=1}^{k} I_{i}(x)=I(x)$ and $\cup_{i=1}^{k} I_{i}(y)=I(y)$ such that $I_{i}(x) \cap I_{j}(x)=\emptyset$ and $I_{i}(y) \cap I_{j}(y)=\emptyset$. From these subintervals, we have $k^{2}$ non-overlapping region such as $I_{i j}(x, y)=I_{i}(x) \times I_{j}(y)$. In each $I_{i j}(x, y)$, if we recursively divide the region, we have $\left(k^{2}\right) n$ subregions for $n \geq 1$. This decomposition derives $k^{2}$-tree description of the image region. Then, the divide and conquer version of our algorithm is described as follows.

\section{Algorithm 2}

1. Divide the imaging region to $\left(k^{2}\right)^{n}$ regions. 
2. Apply Algorithm 1 in each region.

3. Marge the solutions of subregions in the whole region.

4. Set merged data as the initial estimation of the Algorithm $\mathbf{1}$ for whole region.

If there exist $O\left(k^{2}\right)$ ellipses which are not mutually overlapping in the imaging region, approximately, there exist one ellipse in each subregion. For the ellipse and circle fitting problem, approximately there also exists a segment of a ellipse or a ellipse in each subregion. In each region, we can solve the usual model fitting problem for a curve which does not contain data classification process by the permutation process to the data array. Therefore, each subproblem which detects a curve in a subregion is solved faster than the original problem which requires the permutation process to all data array. For a practical application, if an ellipse approximately exists in a $300 \times 300$ pixel subregion of the $1000 \times 1000$ pixel region, we adopt $k=3$ and $n=1$ for the partition of regions which yield 9 subregion in the imaging region.

\section{Numerical Examples}

We estimate the size of the neighborhood on the image plane employing imaging geometry of the pin-hole camera. As usual geometry, we assume that the optical center is at the origin of the world coordinate system, the $z$-axis is the optical axis and that the imaging plane is at $z=f$. Therefore, a point $(x, y, z)^{\top}$ in the space is transformed to $\left(f \frac{x}{z}, f \frac{y}{z}\right)^{\top}$ on the imaging plane. Here, we select the focal length $f$, the imaging area $l \times l$, the number of pixels $L \times L$ as $6 \mathrm{~mm}$, $512 \times 512 \mathrm{~mm}$, and $1024 \times 1024$, respectively.

If the neighborhood of each point is the desk whose radius is $5 \mathrm{~mm}$ on the plane at $z=1000 \mathrm{~mm}$, the neighborhood of each point on the imaging plane is $0.03 \mathrm{~mm}$ which is equivalent to 6 pixels if the resolution of CCD is $0.005 \mathrm{~cm}$. The length $a$ such that $15 \mathrm{~mm} \leq a \leq 25 \mathrm{~mm}$ on the plane at $z=1000 \mathrm{~mm}$ is transformed to $180 \leq a^{\prime} \leq 300$ pixels.

An ellipse $f(x, y)=0$, for $f(x, y)=a x^{2}+2 b x y+c y^{2}+2 d x+2 e y+f$, is expressed as

$$
(\boldsymbol{U}(\boldsymbol{x}-\boldsymbol{a}))^{\top}\left(\begin{array}{cc}
l_{1}^{-1} & 0 \\
0 & l_{2}^{-1}
\end{array}\right)(\boldsymbol{U}(\boldsymbol{x}-\boldsymbol{a}))=0, \boldsymbol{U}=\left(\begin{array}{cc}
\cos \theta & \sin \theta \\
-\sin \theta & \cos \theta
\end{array}\right), \boldsymbol{a}=\left(\begin{array}{l}
p \\
q
\end{array}\right),
$$

where $\boldsymbol{a}, l_{1}, l_{2}$, and $\theta$ are the center of an ellipse, the length of the major axis, the length of the minor axis, and the angle between the major axis and the $x$-axis of the coordinate system. Parameters $k_{1}=\frac{\sqrt{(b q+d)^{2}-4\left(b q^{2}+e q+f\right)}}{2}$ and $k_{2}=$ $\frac{\sqrt{(b p+d)^{2}-4\left(a p^{2}+d p+f\right)}}{2}$ are the half of the lengths of line segments defined by $\left\{(x, y)^{\top} \mid f^{2}(x, y)=0, y=q\right\}$ and $\left\{(x, y)^{\top} \mid f(x, y)=0, x=p\right\}$, respectively. Setting $\hat{l}_{i}, \hat{k_{i}}$, for $i=1,2$, and $\hat{\boldsymbol{a}}$ to be the parameters of the reconstructed ellipse, if $\hat{\boldsymbol{a}} \cong \boldsymbol{a}, \hat{l}_{i} \cong l_{i}$ and $\hat{\theta} \cong \theta$, then $\hat{k}_{i} \cong k_{i}$. Furthermore, if $\theta=0$, then $l_{i}=k_{i}$ and if $\theta=\frac{\pi}{2} l_{1}=k_{2}$ and $l_{2}=k_{1}$. Therefore, parameters $\left|\hat{k}_{i}-k_{i}\right|$ 
for $i=1,2$ act as a parameter for the evaluation of the angle between the major axes of two ellipses if two ellipses are almost overlapping. From these geometric properties, in this paper, we evaluate $\left|k_{i}-\hat{k_{i}}\right|$.

Setting $r$ to be the radius of the neighborhood of a point on the imaging plane, if the reconstructed ellipse $\hat{f}(x, y)=0$ exists in $\mathbf{B} \oplus \mathbf{D}(r)$ for $\mathbf{B}=$ $\left\{(x, y)^{\top} \mid f(x, y)=0\right\}$ and $\mathbf{D}(r)=\left\{(x, y)^{\top} \mid x^{2}+y^{2} \leq r^{2}\right\}$, parameters $\hat{l}_{i}$ and $\hat{k}_{i}$, and vector $\hat{\boldsymbol{a}}$ satisfy the relations $\left|l_{i}-\hat{l}_{i}\right| \leq r,\left|k_{i}-\hat{k}_{i}\right| \leq \sqrt{2} r$, and $|\boldsymbol{a}-\hat{\boldsymbol{a}}| \leq r$. Next, if the neighborhood $\mathbf{D}(r)$ is approximated by 24-neighborhood in $\mathbf{Z}^{2}, r$ is approximated as $\frac{\sqrt{5}}{2}$ pixels.

Next, we define a digital ellipse as a sampling model for the numerical evaluation. Setting $\lambda_{1}(\beta) \leq \lambda_{2}(\beta)$ to be the real solutions of $f(x, \beta)=0$, for $\beta \in \mathbf{Z}$, we define four discrete point sets as

$$
\begin{aligned}
& \mathbf{O}_{1}=\left\{\left(\left\lceil\lambda_{1}(\beta)\right\rceil, \beta\right)^{\top} \mid f(x, \beta)=0\right\}, \mathbf{O}_{2}=\left\{\left(\left\lfloor\lambda_{2}(\beta)\right\rfloor, \beta\right)^{\top} \mid f(x, \beta)=0\right\} \\
& \mathbf{O}_{3}=\left\{\left(\left\lfloor\lambda_{1}(\beta)\right\rfloor, \beta\right)^{\top} \mid f(x, \beta)=0\right\}, \mathbf{O}_{4}=\left\{\left(\left\lceil\lambda_{2}(\beta)\right\rceil, \beta\right)^{\top} \mid f(x, \beta)=0\right\} .
\end{aligned}
$$

With the same manner, setting $\mu_{1}(\alpha) \leq \mu_{2}(\alpha)$ to be the real solutions of $f(\alpha, y)=0$, for $\alpha \in \mathbf{Z}$, we define four discrete point sets as

$$
\begin{aligned}
& \mathbf{O}_{5}=\left\{\left(\alpha,\left\lceil\mu_{1}(\alpha)\right\rceil\right)^{\top} \mid f(\alpha, y)=0\right\}, \mathbf{O}_{6}=\left\{\left(\alpha,\left\lfloor\mu_{2}(\alpha)\right\rfloor\right)^{\top} \mid f(\alpha, y)=0\right\} \\
& \mathbf{O}_{7}=\left\{\left(\alpha,\left\lfloor\mu_{1}(\alpha)\right\rfloor\right)^{\top} \mid f(\alpha, y)=0\right\}, \mathbf{O}_{8}=\left\{\left(\alpha,\left\lceil\mu_{2}(\alpha)\right\rceil\right)^{\top} \mid f(\alpha, y)=0\right\} .
\end{aligned}
$$

We adopt $\mathbf{O}=\cup_{i=1} \mathbf{O}_{i}$ as the discrete ellipse derived from $f(x, y)=0$. Furthermore, setting $\mathbf{R}_{24}$ to be randomly selected points in $\mathbf{N}_{24}=\left\{\boldsymbol{x}=(x, y)^{\top} \mid x^{2}+\right.$ $\left.y^{2} \leq 8, \boldsymbol{x} \in \mathbf{Z}^{2}\right\}$, we adopt $\mathbf{E}=(\mathbf{O} \oplus \mathbf{R}) \backslash \mathbf{O}$ as the collection of discrete sample points from ellipse $f(x, y)=$.

We have evaluated the averages and averages variances of $P=|\boldsymbol{a}-\hat{\boldsymbol{a}}|, L_{i}=$ $\left|l_{i}-\hat{l}_{i}\right|$, and $K_{i}=\left|k_{i}-\hat{k}_{i}\right|$ for 10 images in each group. We express the average and average of variance of each parameter as $e_{E(\cdot)}$ and $e_{V(\cdot)}$. For each group, we generated 5 ellipses and the region of interest is separated into 9 regions. In tables, we list the values $a$ and $b$ which determine the density of sample points and signal-to-noise ratio. We set $a=10,50,100$, and $b=0,20 a / 100$ is the ratio of the selected sample points from discrete approximation of each ellipse. Furthermore $b / 100$ is the ratio of random noise in the background. Table 1 shows the figures for evaluation and Table 2 shows the computational times for each group. Figures 1 (a) and 1 (b) show a noisy image of ellipses for $a=10$ and $b=20$.

If we set $\boldsymbol{\xi}=(x, y, 1)^{\top}$, which is equivalent to set $a=b=c=0$ in eq. (1), the method also detects lines. Therefore, we apply the method for the detection of conics and lines which exist in an image. We first detect lines, since for the detection of lines sample points lie on conics affect as background noise during line detection. After detecting lines, we apply the ellipse-detection algorithm. The endpoints of line segment and parts of ellipses are detected back-voting lines and ellipes to the image plane. We extract back-voted lines and parts of ellipses which lie in the union of the neighborhoods of sample points on the imaging plane. Figures 2 (a), and 2 (b) show detected lines and ellipse from an 
Table 1. Computational results of fitting of ellipses

\begin{tabular}{|r|r|r|r|r|r|r|}
\hline Group & 1 & 2 & 3 & 4 & 5 & 6 \\
\hline$a$ & 100 & 50 & 10 & 100 & 50 & 10 \\
\hline$b$ & 0 & 0 & 0 & 20 & 20 & 20 \\
\hline$e_{E\left(L_{1}\right)}$ & 0.036024 & 0.047197 & 0.129199 & 0.033195 & 0.039478 & 0.099252 \\
\hline$e_{E\left(L_{2}\right)}$ & 0.039044 & 0.051161 & 0.100132 & 0.026181 & 0.040938 & 0.112451 \\
\hline$e_{E\left(K_{1}\right)}$ & 0.047152 & 0.060962 & 0.164751 & 0.046178 & 0.056796 & 0.138115 \\
\hline$e_{E\left(K_{2}\right)}$ & 0.048523 & 0.052492 & 0.105435 & 0.027857 & 0.039804 & 0.090192 \\
\hline$\left.e_{E(P)}\right)$ & 0.048479 & 0.068985 & 0.167061 & 0.029707 & 0.050235 & 0.129238 \\
\hline$e_{V\left(L_{1}\right)}$ & 0.000904 & 0.001432 & 0.027780 & 0.000760 & 0.001184 & 0.003777 \\
\hline$e_{V\left(L_{2}\right)}$ & 0.001621 & 0.002185 & 0.005338 & 0.000500 & 0.000876 & 0.007313 \\
\hline$e_{V\left(K_{1}\right)}$ & 0.004803 & 0.005625 & 0.035757 & 0.001111 & 0.001723 & 0.010337 \\
\hline$e_{V\left(K_{2}\right)}$ & 0.003622 & 0.003567 & 0.006834 & 0.000457 & 0.001519 & 0.004738 \\
\hline$e_{V(P)}$ & 0.002581 & 0.003924 & 0.015863 & 0.015863 & 0.000705 & 0.004161 \\
\hline
\end{tabular}

Table 2. Computational times for each group

\begin{tabular}{|r|r|r|r|r|r|r|}
\hline Group & 1 & 2 & 3 & 4 & 5 & 6 \\
\hline$a$ & 100 & 50 & 10 & 100 & 50 & 10 \\
\hline$b$ & 0 & 0 & 0 & 20 & 20 & 20 \\
\hline time(s) & 34.72 & 21.41 & 10.71 & 146.67 & 97.89 & 81.94 \\
\hline
\end{tabular}

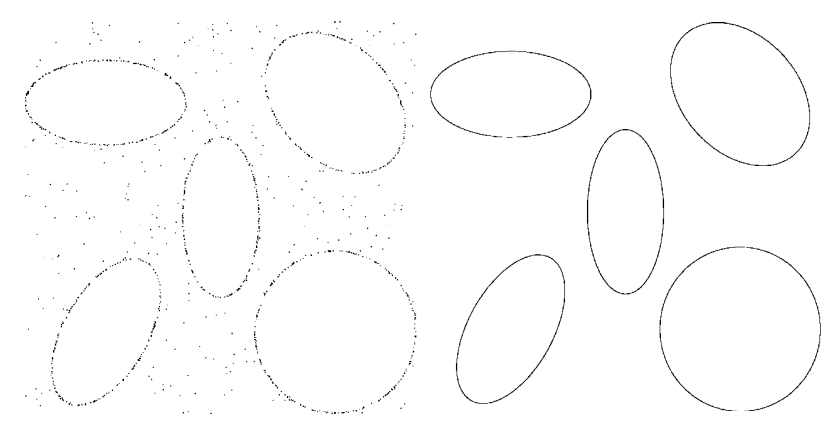

(a)

(b)

Fig. 1. (a)Ellipses with background noise, and (b) detected ellipses 
image with a house and a cylinder, and detected line-segments and elliptic arcs from an image, respectively. These results for synthetic data and a real image show that our method effectively detects lines and conics in an image without using any acumulators.

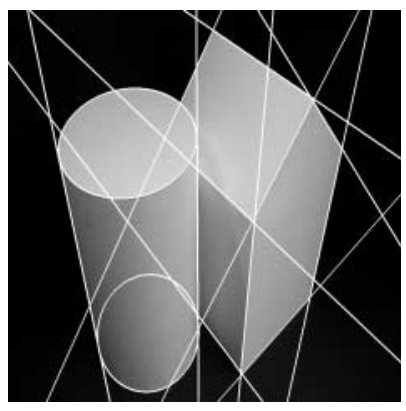

(a)

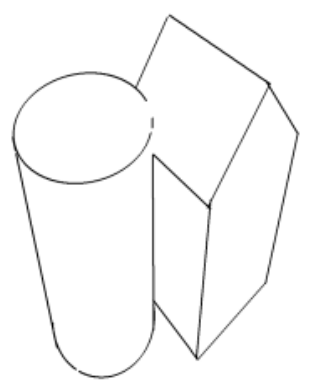

(b)

Fig. 2. (a) Detected lines and ellipes from an image with a house and a cylinder, (b) Detected line-segments and elliptic arcs from an image with a house and a cylinder

\section{Conclusions}

We introduced the combinatorial Hough transform which is the Hough transform without any accumulators. The combinatorial Hough transform is based on the mathematical property that the grouping of sample points is achieved by the permutation for the sequence of the sample points. We also showed the convergence of the algorithm deriving a dynamic system which achieves the minimization of the criterion for the detection of figures from unclassified sample points.

\section{References}

1. Ballard, D. and Brown, Ch. M., Computer Vision, Prentice-Hall; New Jersey, 1982. 823

2. Deans, S. R., Hough transform from the Radon transform, IEEE Trans. Pattern Analysis and Machine Intelligence, PAMI-3, 185-188, 1981. 823

3. Levers, V. F., Which Hough transform? CVGIP: Image Understanding, 58, 250264, 1993. 823 
4. Becker, J.-M., Grousson, S., and Guieu, D., Space of circles: its application in image processing, Vision Geometry IX, Proceedings of SPIE, 4117, 243-250, 2000. 823

5. Cox, D., Little, J., and O'Shea, D., Ideals, Varieties, and Algorithms: An Introduction to Computational Algebraic Geometry and Commutative Algebra, SpringerVerlag; New York, 1992. 824

6. Mattavelli, M., Noel, V., and Ammaldi, E., Fast line detection algorithms based on combinatorial optimization, LNCS, 2051, 410-419, 2001. 825

7. Brockett, R. W., Least square matching problem, Linear Algebra and its Applications, 122/123/124, 1989, 761-777. 826

8. Brockett, R. W., Dynamical system that sort list, diagonalize matrices, and solve linear programming problems, Linear Algebra and its Applications, 146, 1991, 7991. 826

9. Vandenberghe, L. and Boyd, S., Semdefnite programming, SIAM Review, 38, 4995, 1996. 827

10. Alizaden, F., Interir point methods in semidefinite programming with application to combinatorial optimization, SIAM, Journal on Optimization, 5, 13-51, 1995. 827 